\title{
Genome Mining of Rice (Oryza sativa subsp. indica) for Detection and Characterization of Long Palindromic Sequences
}

\author{
Elmira Katanchi Kheiavi ${ }^{1}$, Asadollah Ahmadikhah ${ }^{2 *}$ and Ali Mohammadian Mosammam ${ }^{3}$ \\ ${ }^{1}$ Department of Agronomy and Plant Breeding, Faculty of Agriculture, University of Zanjan, Zanjan, Iran \\ ${ }^{2}$ Department of Biotechnology, Shahid Beheshti University, Faculty of New Technologies and Energy Engineering, Tehran, Iran \\ ${ }^{3}$ Department of Statistics, Faculty of Science, University of Zanjan, Zanjan, Iran
}

"Corresponding author: Asadollah Ahmadikhah, Department of Biotechnology, Shahid Beheshti University, Faculty of New Technologies and Energy Engineering, Tehran, Iran, E-mail: a_ahmadikhah@sbu.ac.ir

Received date: March 06, 2016; Accepted date: May 24, 2016; Published date: May 30, 2016

Copyright: ( 2016 Kheiavi EK et al. This is an open-access article distributed under the terms of the Creative Commons Attribution License, which permits unrestricted use, distribution, and reproduction in any medium, provided the original author and source are credited.

\begin{abstract}
Because the rice genome has been sequenced entirely, search to find specific features at genome-wide scale is of high importance for studying genome evolution and subsequent applications. Palindromic sequences are important DNA motifs involved in the regulation of different cellular processes and are a potential source of genetic instability. A genome mining approach was applied to detect and characterize the long palindromic sequences in the rice genome. All palindromes, defined as identical inverted repeats with spacer DNA, could be analyzed and sorted according to their frequency, size, GC content, compact index etc. The results showed that the overall palindrome frequency is high in rice genome (nearly 51000 palindromes), that totally cover $41.4 \%$ of nuclear genome of rice, with highest and lowest number of palindromes, respectively belongs to chromosome 1 and 12 . Palindrome number could well explain the rice chromosome expansion (R2>92\%). Average GC content of the palindromic sequences is $42.1 \%$, indicating AT-richness and hence, the low-complexity of palindromic sequences. The results also showed different compact indices of palindromes in different chromosomes (43.2 per cM in chromosome 8 and 34.5 per cM in chromosome 3, as highest and lowest, respectively). Co-location analysis showed that more than $20 \%$ of rice genes overlapped with palindromic regions, mainly concentrating on chromosomal arms. Based on the results of this research it can be concluded that the rice genome is rich in long palindromic sequences that triggered most variation during evolution. Generally, both sections of palindromic sequences including stems and loops are AT-rich, indicating that these regions locate in the low-complexity segments of the rice chromosomes.
\end{abstract}

Keywords: Genome mining; Low-complexity; Palindrome; Repetitive; Rice; R programming
Abbreviations
CI: Compactness Index; CL: Chromosome Length; DSB: Double- Strand Break; MITE: Miniature Inverted Transposable Element; PL: Palindrome Length; TE: Transposable Element

\section{Introduction}
Rice is one of the most important cereal crops around the world having the smallest genome among the cereals [1]. Rice is considered as a suitable model in genetic studies of cereal crops and other grasses. Repetitive sequences in rice genome are classified into transposable elements (TEs), sequences associated with centromere, telomeres, rRNA-related genes and other unknown sequences. Some of these repetitive sequences have known biological roles such as rRNA genes, centromeres and telomeres, while the role of other repetitive sequences

stayed unknown [2]. Repetitive sequences significantly represent a candidate for regulatory sites [3]. The main class of repetitive sequences are palindrome regions in different genomes. Palindromes are present in both prokaryotes and eukaryotes and they involve in many biological processes, for example they act as recognition sites for activity of restriction enzymes, play an important role in DNA replication and regulation of gene expression through the formation of stem-loop structure [4].

Palindrome-containing sequences in mouse, yeast and bacteria genomes show a high instability. Inverted repeats of $<10 \mathrm{bp}$ distance cause B-DNA to form a cruciform structure [5]. Short palindrome sequences $(60-66 \mathrm{bp})$ named palindrome repeat sequences (PRS) were found in the rice genome. A single PRS was found in the intron region of mitochondrial gene rps3 (ribosomal protein 3) in rice [6]. Study on distribution pattern of palindromes in yeast (Saccaromyses cervisiea) genome showed that long palindromes are rich in AT and mainly locate in intron regions of genes [7]. A representative scheme showing the inverted repeat sequences in yeast is shown in Figure 1. 
Citation: Kheiavi EK, Ahmadikhah A, Mosammam AM (2016) Genome Mining of Rice (Oryza sativa ssp. indica) for Detection and

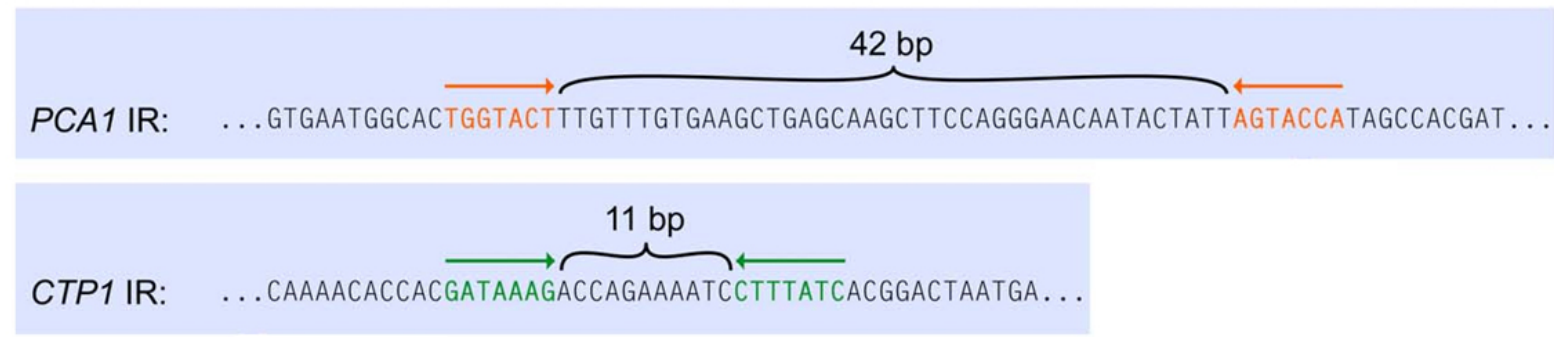

Figure 1: The inverted repeat sequences in CTP1 and PCA1 genes that define the breakpoints of a specific SUL1 amplification event in yeast [8]. Adopted from Brewer et al. [9].

Pattern analysis of tandem repeats in genes is an indispensable computational approach to understand the gene expression and pathogenesis of diseases in humans [10]. The high mutation rate in tandem repeats is primarily due to the error in DNA replication that leads to the changes in the number of repeat units. In addition, it is also known that variable tandem repeats in the genome of an organism can accelerate the evolution of coding and regulatory gene sequences [11]. The information obtained from the investigation of tandem repeats has widespread application in various fields such as medicine, forensic science, and population genetics [12]. Notably, the identification of tandem repeats has special function in genetic studies, such as gene mapping [13]. Tandem repeats are a stretch of nucleotides that repeat in a consecutive manner. They are ubiquitous in genome and their mutated forms are responsible for diseases [11]. Understanding of the patterns of tandem repeats may provide insights into genome evolution and gene expression patterns. The advancement of computational tools such as computer algorithms, databases and web servers provide an efficient avenue to analyze enormous volume of genetic data, such as tandem repeats in large genome.

Location of these elements inside or near the active genes causes to start or end of the gene activity. Selection of new genes are produced looking for mutation or gene integration, requires forces that will determine the evolution of organisms [1-17]. Identification of repetitive regions provides insight into expression patterns of disease genes. Development of computational tools such as computer algorithms, databases and web servers, has made it possible to develop in silico approaches suitable for analysis of genetic data, including constituent repetitive sequences in the entire genome. $\mathrm{R}$ is both a language and an interface for statistical analysis, programming, and graphics. $\mathrm{R}$ is modeled after the $\mathrm{S}$ language that was originally created by AT\&T and in many cases scripts written for $\mathrm{R}$ can be run in $\mathrm{S}$ with little to no modification. $\mathrm{R}$ has become a standard interface for statistical analysis in biological sciences due in part to its openness, ability to be extended by users and it vibrant user base [18].

Regarding that the statistical programming language $\mathrm{R}$ has become a de facto standard for the analysis of many types of biological data [19], we attempted to utilize this programming language for identifying long palindrome sequences in the rice ( $O$. sativa subsp. indica) genome, and also for extracting some of their specific features including GC content, nucleotide composition of the detected palindrome sequences in each rice chromosome, and for finding the overlapping between the detected palindromes and genic regions.

\section{Material and Methods}

\section{Data sets and palindrome extraction}

At first step, the genomic sequences of 12 chromosomes of rice (Oryza sativa subsp. indica) were retrieved from NCBI website (www.ncbi.nlm.nih.gov) and stored in a PC drive in "FASTA" format to create early data set. FASTA files were called in R, and "Biostrings" package and "findPalindrome" command were used to extract palindromic regions in each rice chromosome. The criteria for extracting palindromic regions were as follow: Minimum arm length $=20$ nucleotides, minimum loop length $=0$ nucleotides (because existed available "findPalindrome" command doesn 't permits other numbers), maximum loop length $=10000$ nucleotides and allowing no mismatch (maximum mismatch $=0$ ). The $\mathrm{R}$ script is as following:

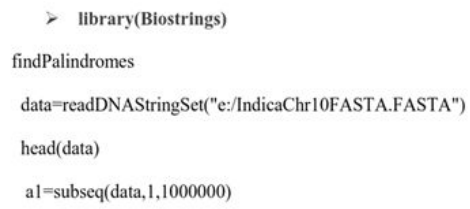

A Perl programme was used to call the results of $\mathrm{R}$ programme. Secondary commands were written to filter out unwanted results such as very short palindromes (by setting minimum loop length $=60$ nucleotides). Final outputs were stored to create secondary data set. GC content of each palindrome region (partitioned in stem, loop and total) was calculated using "Biostrings" and "seqinr" packages. The R script is as following:

Frequencies of palindromic sequences were separately calculated for each chromosome in one million intervals. Compactness index (CI) in view of the number of palindromes in each interval and in whole chromosome was calculated as the number of palindromes per Mbp and or per $\mathrm{cM}$ (given that $1 \mathrm{cM}=280 \mathrm{Kbp}$ in rice genome).

To determine overlapping of detected palindromes with genic regions, the physical locations of all genes in the rice genome were taken from (http://natural.salk.edu/databases/RiceiGE) in "txt" format to create 3rd data set. The overlaps between palindromes and genes were obtained using "IRanges" package and "findOverlap" command. The R script is as following: 
Citation: Kheiavi EK, Ahmadikhah A, Mosammam AM (2016) Genome Mining of Rice (Oryza sativa ssp. indica) for Detection and

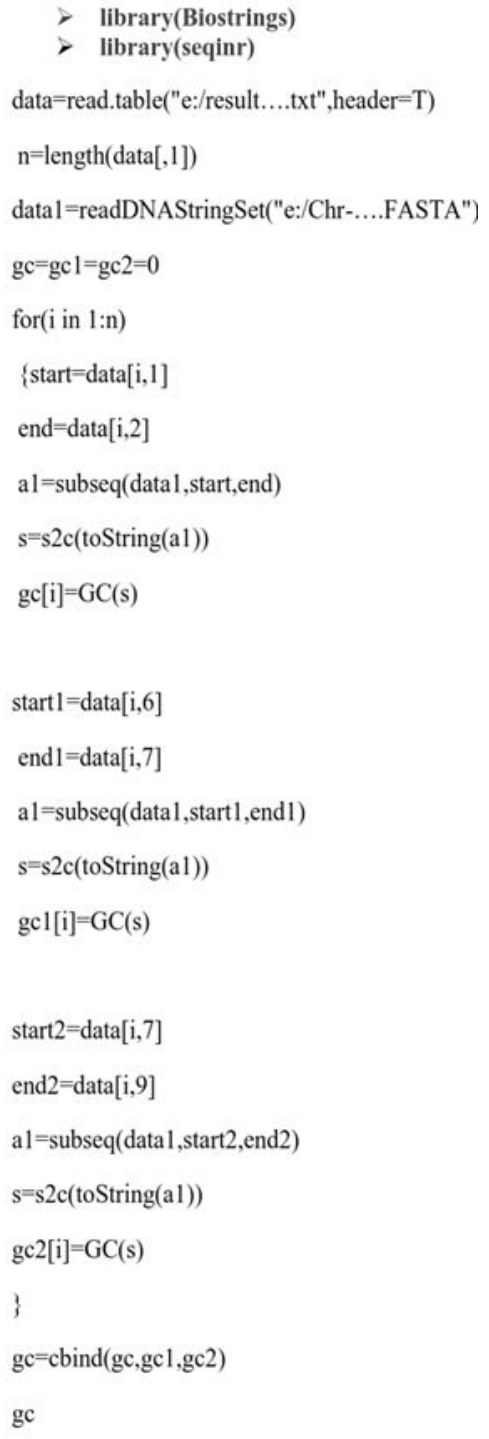

\section{Statistical Analysis}

Microsoft Office Excel 2007 was used to perform regression analysis to predict correlation between genome size, palindrome size and abundance. Graphical presentations were conducted in excel worksheet.

\section{Results}

\section{Palindrome frequency}

Searching for palindromic regions in the rice genome showed that rice genome has high frequency of palindromic regions (Additional File 1). In total, with our searching criteria, rice genome has nearly 51000 long palindrome sequences, with an average of 4243 palindrome regions per chromosome (Table 1, Figure 2). Chromosome 1 has the highest (6598) and chromosome 12 has the least (2993) number of palindromes. Palindrome regions totally cover $41.4 \%$ of nuclear genome of rice, with highest coverage (52.9\%) for chromosome 10 and lowest coverage $(32.3 \%)$ for chromosome 3. For more details see Additional File 2.
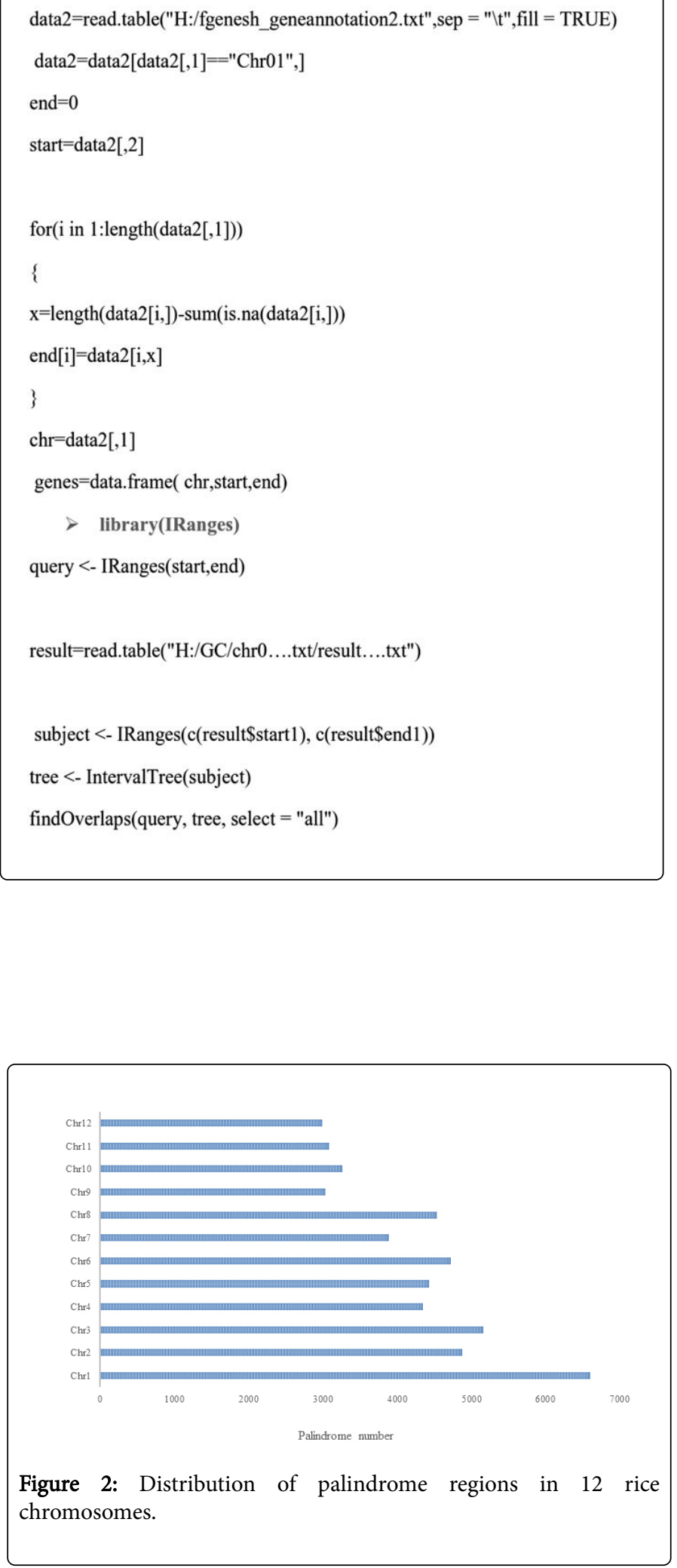
Citation: Kheiavi EK, Ahmadikhah A, Mosammam AM (2016) Genome Mining of Rice (Oryza sativa ssp. indica) for Detection and Characterization of Long Palindromic Sequences. J Data Mining Genomics \& Proteomics 7: 199. doi:10.4172/2153-0602.1000199

Page 4 of 10

\begin{tabular}{|c|c|c|c|c|c|c|}
\hline Chromosome & $\begin{array}{l}\text { Chromosome } \\
\text { length (Kbp) }\end{array}$ & $\begin{array}{l}\text { No. } \\
\text { palindrome of } \\
\text { regions }\end{array}$ & $\begin{array}{l}\text { Chromosome coverage } \\
(\%)\end{array}$ & $\begin{array}{l}\text { Average } \\
\text { palindrome } \\
\text { length (bp) }\end{array}$ & $\begin{array}{l}\text { Average } \\
\text { length (bp) }\end{array}$ & $\begin{array}{l}\text { Average loop length } \\
\text { (bp) }\end{array}$ \\
\hline 1 & 47244.9 & 6598 & $19524.7(41.3 \%)$ & $2959.2(104-10792)$ & $33.9(20-1610)$ & 2891.4 (61-9991) \\
\hline 2 & 38080.4 & 4873 & $13234.4(34.8 \%)$ & $2715.9(101-10451)$ & $32.5(20-1585)$ & $2650.8(61-9977)$ \\
\hline 3 & 41835.9 & 5159 & $13511.9(32.3 \%)$ & 2619.1 (102-10099) & $32.7(20-867)$ & $2553.7(61-9988)$ \\
\hline 4 & 34660.7 & 4341 & $12903.7(37.2 \%)$ & $2972.5(102-10988)$ & $33.4(20-1165)$ & $2905.6(61-10000)$ \\
\hline 5 & 31162.6 & 4430 & $13882.6(44.5 \%)$ & $3133.8(101-10134)$ & $33.2(20-767)$ & 3068.4 (61-9999) \\
\hline 6 & 32845.4 & 4719 & $14631.1(44.5 \%)$ & $3100.5(102-10205)$ & $33.6(20-942)$ & $3033.3(62-9996)$ \\
\hline 7 & 27898.3 & 3886 & $11584.3(41.5 \%)$ & $2981.0(101-10137)$ & $31.7(20-989)$ & $2917.6(61-9993)$ \\
\hline 8 & 29367.1 & 4535 & $14496.0(49.4 \%)$ & $3196.5(102-10167)$ & $35.4(20-1104)$ & $3125.7(61-9993)$ \\
\hline 9 & 21712.9 & 3031 & $9431.7(43.4 \%)$ & $3111.8(101-11574)$ & $34.1(20-1203)$ & $3043.5(61-9998)$ \\
\hline 10 & 22150.1 & 3261 & $11726.9(52.9 \%)$ & $3596.1(102-10244)$ & $34.1(20-1418)$ & $3527.8(61-9990)$ \\
\hline 11 & 22971.7 & 3088 & $9864.3(42.9 \%)$ & $3194.4(102-10259)$ & $33.0(20-1080)$ & $3128.4(61-9994)$ \\
\hline 12 & 22948.0 & 2993 & $9450.9(41.2 \%)$ & $3157.7(102-11061)$ & $33.0(20-1337)$ & $3091.6(61-9979)$ \\
\hline Total/mean & 372887.9 & 50914 & $154242.5(41.4 \%)$ & $3029.5(101-11574)$ & $33.4(20-1610)$ & $2962.8(61-10000)$ \\
\hline
\end{tabular}

Table 1: Number of palindrome regions in 12 indica rice chromosomes and some related statistics.

Average whole palindrome length is 3029 bp ranging from 101 to $11574 \mathrm{bp}$ (Figure 3). The highest average palindrome length (3596 bp) belongs to chromosome 10 and the lowest (2619 bp) belongs to chromosome 3. Average stem length is $33.4 \mathrm{bp}$ ranging from 20 to 1610 bp. The highest average stem length $(35.4 \mathrm{bp})$ belongs to chromosome
8 and the lowest (31.7 bp) belongs to chromosome 7. Average loop length is nearly $2963 \mathrm{bp}$ ranging from 61 to $10000 \mathrm{bp}$. The highest average loop length (3527 bp) belongs to chromosome 10 and the lowest (2553 bp) belongs to chromosome 3.
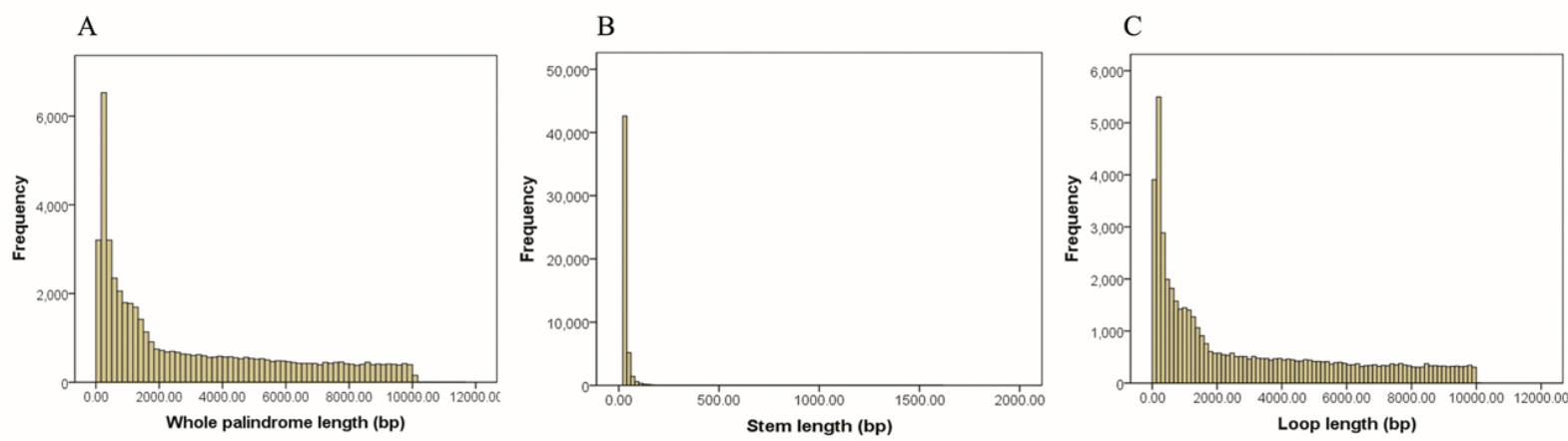

Figure 3: Frequency distribution of whole palindrome length (A), stem length (B) and loop length (C) for detected palindrome regions in rice nuclear genome (for more details see Additional File 3). 
Citation: Kheiavi EK, Ahmadikhah A, Mosammam AM (2016) Genome Mining of Rice (Oryza sativa ssp. indica) for Detection and Characterization of Long Palindromic Sequences. J Data Mining Genomics \& Proteomics 7: 199. doi:10.4172/2153-0602.1000199

Page 5 of 10

Correlation analysis showed that palindrome number has a high positive effect on chromosome expansion (R2>92.5\%); in contrast palindrome length has a relatively high negative effect on chromosome expansion $(\mathrm{R} 2>51 \%)$ that it can be explained by the size of loop section (and not by that of stem section) of palindrome regions (Figure 4).
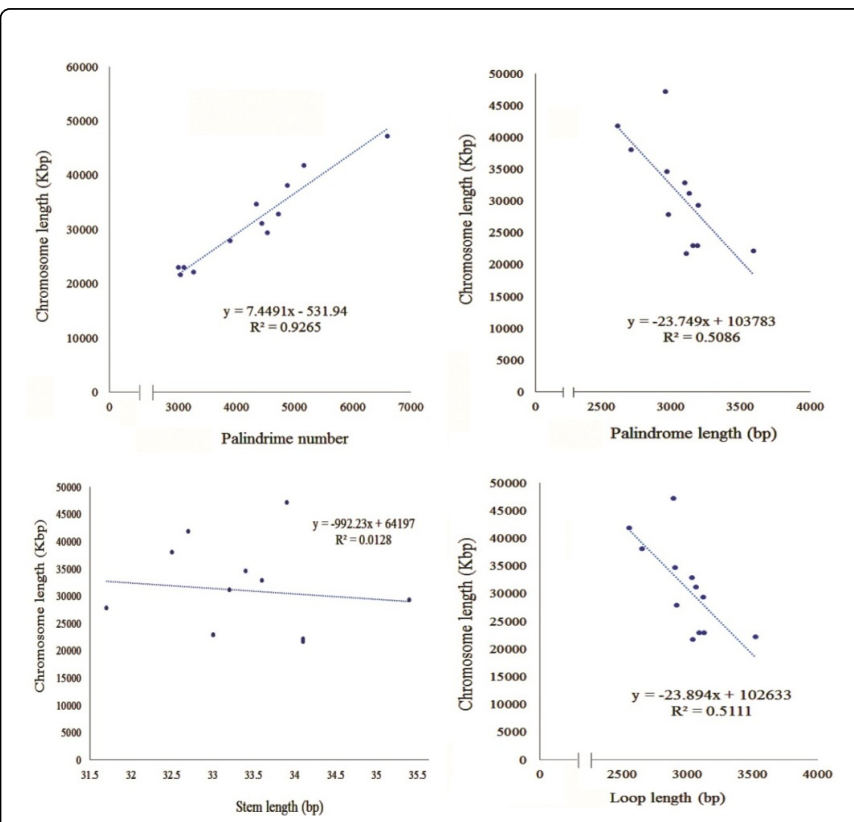

Figure 4: Effect of palindrome number, palindrome length, stem length and loop length on rice chromosome expansion.

\section{GC content}

Average GC contents of palindrome regions in 12 chromosomes are depicted in Table 2. As seen, average of whole GC contents of the palindromes in rice genome is $42.1 \%$, ranging from $41.0 \%$ (in chromosome 11) to $43.3 \%$ (in chromosome 5). The highest stem GC content $(42.6 \%)$ was calculated for chromosome 10 and the lowest (40.0\%) for chromosomes 11 and 12. The highest loop GC content (43.2\%) was calculated for chromosome 5 and the lowest (40.8\%) for chromosome 11. Based on these results, it seems that average GC contents of loops are slightly more than that of stems ( $42.0 \%$ vs. $40.9 \%$ in average). A graph showing stem and loop GC contents of palindrome regions of 12 chromosomes is presented in Figure 5.

More detailed analysis on the abundance and frequency of GC-rich palindrome sequences (Table 3) showed that 6824 (13.4\%) and 4316 (8.5\%) of the detected palindromes (out of 50914) are GC-rich in stems and loops sections, respectively. In contrast, 26516 (52.1\%) and 20087 (39.5\%) of palindromes are AT-rich in stems and loops sections, respectively.

\begin{tabular}{|l|l|l|l|}
\hline Chromosome & Stems GC & Loops GC & $\begin{array}{l}\text { Whole GC (stems } \\
+ \text { loops) }\end{array}$ \\
\hline 1 & $40.7( \pm 16.9)$ & $42.2( \pm 12.2)$ & $42.3( \pm 11.6)$ \\
\hline 2 & $40.3( \pm 16.4)$ & $42.0( \pm 12.6)$ & $42.1( \pm 11.9)$ \\
\hline 3 & $40.9( \pm 11.9)$ & $42.0( \pm 16.9)$ & $42.2( \pm 12.6)$ \\
\hline 4 & $41.0( \pm 11.4)$ & $41.6( \pm 16.7)$ & $41.70( \pm 12.0)$ \\
\hline 5 & $41.2( \pm 11.5)$ & $43.2( \pm 16.6)$ & $43.3( \pm 12.1)$ \\
\hline 6 & $41.1( \pm 11.8)$ & $42.6( \pm 17.2)$ & $42.7( \pm 12.4)$ \\
\hline 7 & $41.3( \pm 11.5)$ & $42.0( \pm 17.7)$ & $42.1( \pm 12.0)$ \\
\hline 8 & $40.2( \pm 11.0)$ & $41.5(16.4)$ & $41.6( \pm 11.5)$ \\
\hline 9 & $41.3( \pm 11.2)$ & $41.5( \pm 16.9)$ & $41.6( \pm 11.8)$ \\
\hline 10 & $42.6( \pm 8.4)$ & $42.4( \pm 16.1)$ & $42.6( \pm 9.0)$ \\
\hline 11 & $40.0( \pm 11.2)$ & $40.8( \pm 16.5)$ & $41.0( \pm 11.9)$ \\
\hline 12 & $40.0( \pm 11.0)$ & $41.0( \pm 16.1)$ & $41.1( \pm 11.8)$ \\
\hline Grand mean & $40.9( \pm 16.8)$ & $42.0( \pm 12.0)$ & $42.1( \pm 11.3)$ \\
\hline & & & \\
\hline
\end{tabular}

Table 2: Average GC content of all palindrome regions for 12 indica rice chromosomes. Standard deviations are shown in parenthesis.

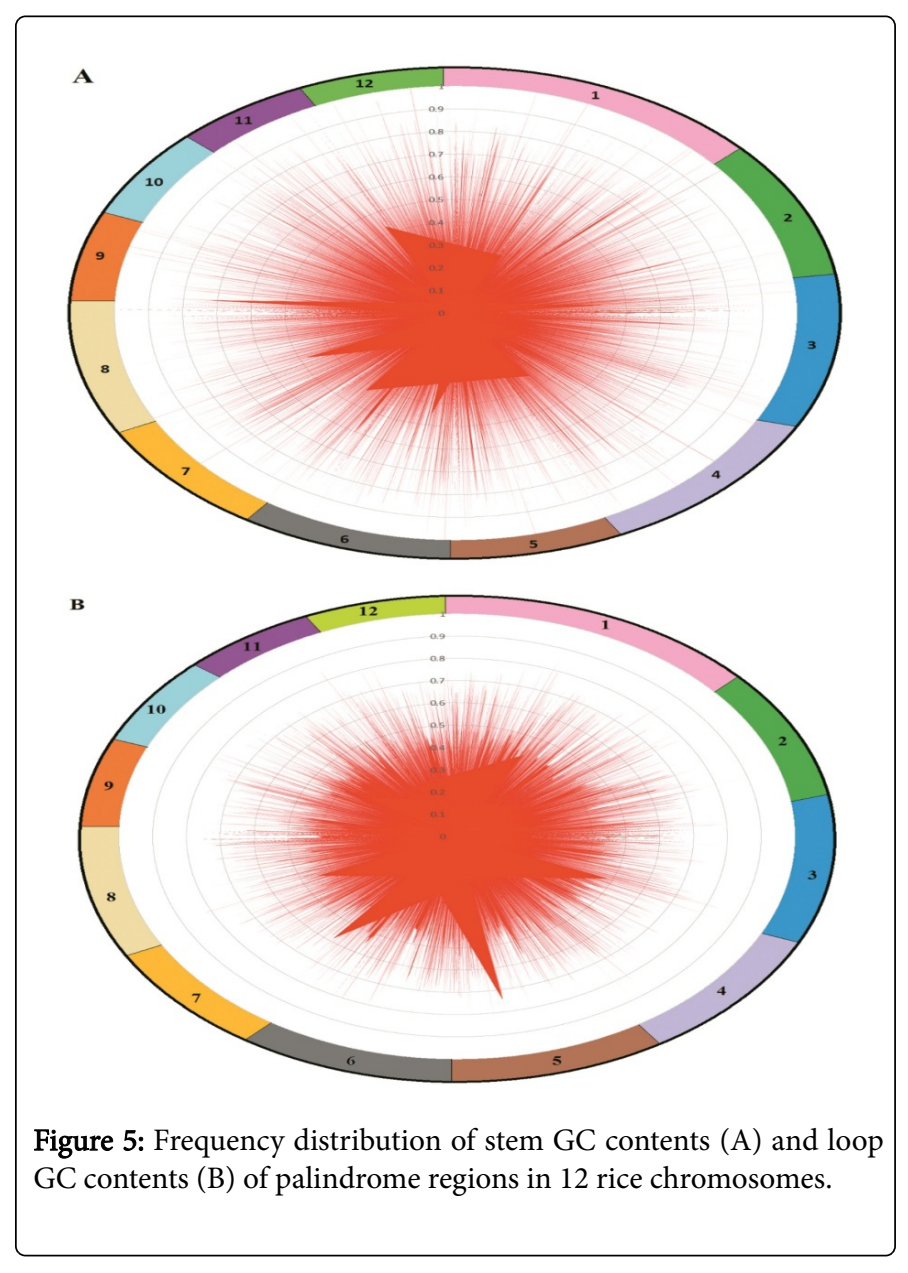


Citation: Kheiavi EK, Ahmadikhah A, Mosammam AM (2016) Genome Mining of Rice (Oryza sativa ssp. indica) for Detection and

Page 6 of 10

\begin{tabular}{|c|c|c|c|c|c|c|c|c|}
\hline \multirow[t]{3}{*}{ Chromosome } & \multicolumn{4}{|c|}{ Stems } & \multicolumn{4}{|c|}{ Loops } \\
\hline & \multicolumn{2}{|c|}{ GC-rich } & \multicolumn{2}{|c|}{ AT-Rich } & \multicolumn{2}{|c|}{ GC-rich } & \multicolumn{2}{|c|}{ AT-Rich } \\
\hline & $n$ & $f(\%)$ & $n$ & $f(\%)$ & $n$ & $f(\%)$ & $n$ & $f(\%)$ \\
\hline 1 & 933 & 14.14 & 3472 & 52.62 & 638 & 9.67 & 2521 & 38.21 \\
\hline 2 & 577 & 11.84 & 2607 & 53.50 & 504 & 10.34 & 2065 & 42.38 \\
\hline 3 & 713 & 13.82 & 2718 & 52.68 & 498 & 9.65 & 2097 & 40.65 \\
\hline 4 & 581 & 13.39 & 2210 & 50.91 & 343 & 7.90 & 1674 & 38.56 \\
\hline 5 & 588 & 13.27 & 2271 & 51.26 & 417 & 9.41 & 1540 & 34.76 \\
\hline 6 & 662 & 14.03 & 2425 & 51.39 & 439 & 9.30 & 1725 & 36.55 \\
\hline 7 & 610 & 15.70 & 2027 & 52.16 & 312 & 8.03 & 1529 & 39.35 \\
\hline 8 & 556 & 12.26 & 2419 & 53.34 & 337 & 7.43 & 1908 & 42.07 \\
\hline 9 & 425 & 14.02 & 1529 & 50.44 & 233 & 7.69 & 1204 & 39.72 \\
\hline 10 & 492 & 15.09 & 1520 & 46.61 & 155 & 4.75 & 1172 & 35.94 \\
\hline 11 & 366 & 11.85 & 1695 & 54.89 & 239 & 7.74 & 1414 & 45.79 \\
\hline 12 & 312 & 10.42 & 1623 & 54.23 & 192 & 6.42 & 1238 & 41.36 \\
\hline Total & 6824 & 13.40 & 26516 & 52.08 & 4316 & 8.48 & 20087 & 39.45 \\
\hline
\end{tabular}

Table 3: Abundance and frequency of GC- and AT-rich palindrome sequences calculated separately for stems and loops sections.

\section{Palindrome occurrence and compact indices in the rice genome}

The results of compact indices (in view of palindromes/Mbp and palindromes/cM) are depicted in Table 4. In view of palindrome number per one $\mathrm{Mbp}$, in average 136.6 palindromes occurred every one $\mathrm{Mbp}$ in the rice genome. Chromosome 8 has the highest $(154.4 \pm 57.3)$ and chromosomes 3 have the lowest $(123.3 \pm 39.6)$ palindrome numbers per Mbp. However, in view of palindrome occurrence per $\mathrm{cM}$, in average 38.2 palindromes per $\mathrm{cM}$ exist in the rice genome (given that $1 \mathrm{cM}$ contains $280 \mathrm{Kbp}$ ). However, different chromosomes have different compact indices, so that chromosome 8 has the highest $\mathrm{CI}$ (43.2 palindromes per $\mathrm{cM}$ ), and chromosome 3 has the least CI (34.5 palindromes per cM), (Figure 6; Additional File 5).

Assessment of the occurrence of palindrome regions in rice genome showed that in average one palindrome region occurs every $7323 \mathrm{bp}$ (Table 4). However, the pattern of this occurrence is very variable between different chromosomes as well as in different regions of a specific chromosome (Figure 6). As seen in the figure, convexities represent a high compactness, indicating that repetitive sequences including palindrome regions play a vital role in heterochromatinization of the rice genome. Among rice chromosome segments, short arms of chromosomes 4 and 9 show a highest compactness. Almost for all rice chromosomes, centromeric regions show highest compactness (occurrence of higher palindromes per $\mathrm{cM}$ ). In contrast, telomeric regions don't follow a general rule of palindrome occurrence. As seen in Figure 6, telomeric side of short arms of chromosomes $3,6,8,11$ and 12 , and telomeric side of long arms of chromosomes 2, 5, 6, 9, 10 and 12 have a low compactness (occurrence of lower palindromes per $\mathrm{cM}$ ).

\section{Overlapping with genes}

Frequency of genic sequences overlapped with palindromic regions were separately calculated for each chromosome. Overlap frequency is shown in Table 5. As seen, chromosome 10 has the highest ratio of overlapping genes $(25.66 \%)$ and chromosome 2 has the least ratio of overlapping genes (493) with palindrome regions in the same chromosome. Totally, 8651 out of 42176 rice genes show overlapping with palindrome regions in the rice genome. In average palindromic regions of each chromosome have overlaps with $20.5 \%$ of the genes in the same chromosome. Pattern of distribution of overlapping genes with palindrome regions is nearly identical in different rice chromosomes (Figure 7), so that in most cases this overlapping is more biased towards chromosomal arms. 
Citation: Kheiavi EK, Ahmadikhah A, Mosammam AM (2016) Genome Mining of Rice (Oryza sativa ssp. indica) for Detection and Characterization of Long Palindromic Sequences. J Data Mining Genomics \& Proteomics 7: 199. doi:10.4172/2153-0602.1000199

Page 7 of 10

\begin{tabular}{|c|c|c|c|c|}
\hline Chromosome & Length (cM) & Palindromes/Mbp & Palindromes/cM) & Occurrence (bp/palindrome) \\
\hline 1 & 168.73 & $139.7( \pm 54.9)$ & $39.1( \pm 14.8)$ & $7160.5( \pm 2612.6)$ \\
\hline 2 & 136.00 & $128.0( \pm 57.3)$ & $35.8( \pm 16.0)$ & $7814.6( \pm 3955.7)$ \\
\hline 3 & 149.41 & $123.3( \pm 39.6)$ & $34.5( \pm 11.2)$ & $8109.3( \pm 2974.7)$ \\
\hline 4 & 123.79 & $125.2( \pm 41.6)$ & $35.1( \pm 11.4)$ & $7984.5( \pm 3080.3)$ \\
\hline 5 & 111.29 & $142.2( \pm 58.5)$ & $39.8( \pm 15.4)$ & $7034.4( \pm 3035.8)$ \\
\hline 6 & 117.300 & $143.7( \pm 61.3)$ & $40.2( \pm 17.0)$ & $6960.2( \pm 3685.8)$ \\
\hline 7 & 99.63 & $139.3( \pm 60.4)$ & $39.0( \pm 17.1)$ & $7179.2( \pm 3221.8)$ \\
\hline 8 & 108.45 & $154.4( \pm 57.3)$ & $43.2( \pm 19.7)$ & $6475.7( \pm 3458.2)$ \\
\hline 9 & 77.54 & $139.6( \pm 51.3)$ & $39.1( \pm 13.9)$ & $7163.6( \pm 2886.9)$ \\
\hline 10 & 79.10 & $147.2( \pm 54.5)$ & $41.2( \pm 13.9)$ & $6792.4( \pm 3131.6)$ \\
\hline 11 & 82.04 & $134.4( \pm 38.4)$ & $37.6( \pm 10.7)$ & $7439.0( \pm 2008.3)$ \\
\hline 12 & 81.95 & $130.4( \pm 60.1)$ & $36.5( \pm 16.8)$ & $7667.2( \pm 2600.3)$ \\
\hline Mean & & $136.6( \pm 53.3)$ & $38.2( \pm 15.1)$ & $7323.9( \pm 3116.9)$ \\
\hline
\end{tabular}

Table 4: Compactness indices (CIs) and occurrence of palindromes in rice genome.

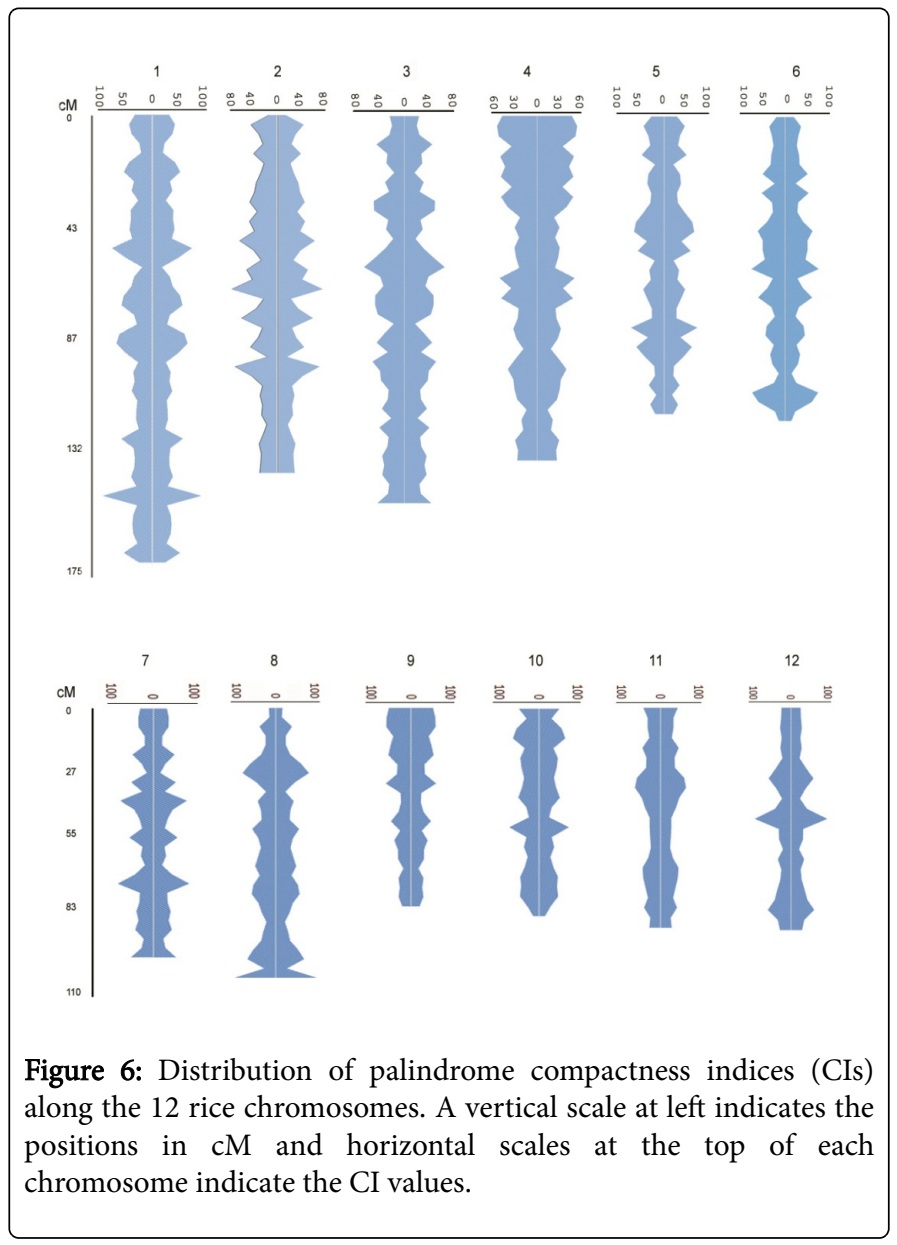

\begin{tabular}{|l|l|l|l|}
\hline Chromosome & $\begin{array}{l}\text { No. } \\
\text { genes }\end{array}$ & $\begin{array}{l}\text { No. of overlapping } \\
\text { genes }\end{array}$ & $\begin{array}{l}\text { Percent of } \\
\text { overlapping genes }\end{array}$ \\
\hline 1 & 5729 & $1144( \pm 8.83)$ & 19.97 \\
\hline 2 & 4528 & $844( \pm 6.56)$ & 18.64 \\
\hline 3 & 4993 & $992( \pm 10.33)$ & 19.87 \\
\hline 4 & 3800 & $722( \pm 7.64)$ & 19.00 \\
\hline 5 & 3435 & $747( \pm 7.85)$ & 21.75 \\
\hline 6 & 3584 & $715( \pm 8.66)$ & 19.95 \\
\hline 7 & 3220 & $686( \pm 8.19)$ & 21.30 \\
\hline 8 & 3131 & $624( \pm 8.66)$ & 19.93 \\
\hline 9 & 2417 & $543( \pm 7.94)$ & 22.47 \\
\hline 10 & 2463 & $632( \pm 16.15)$ & 25.66 \\
\hline 11 & 2525 & $509( \pm 8.81)$ & 20.16 \\
\hline 12 & 2351 & $493( \pm 8.73)$ & 20.97 \\
\hline Total & 42176 & $8651( \pm 9.13)$ & 20.51 \\
\hline & & & \\
\hline
\end{tabular}

Table 5: Frequency of genic sequences overlapped with palindromic regions in 12 rice chromosomes. 
Citation: Kheiavi EK, Ahmadikhah A, Mosammam AM (2016) Genome Mining of Rice (Oryza sativa ssp. indica) for Detection and
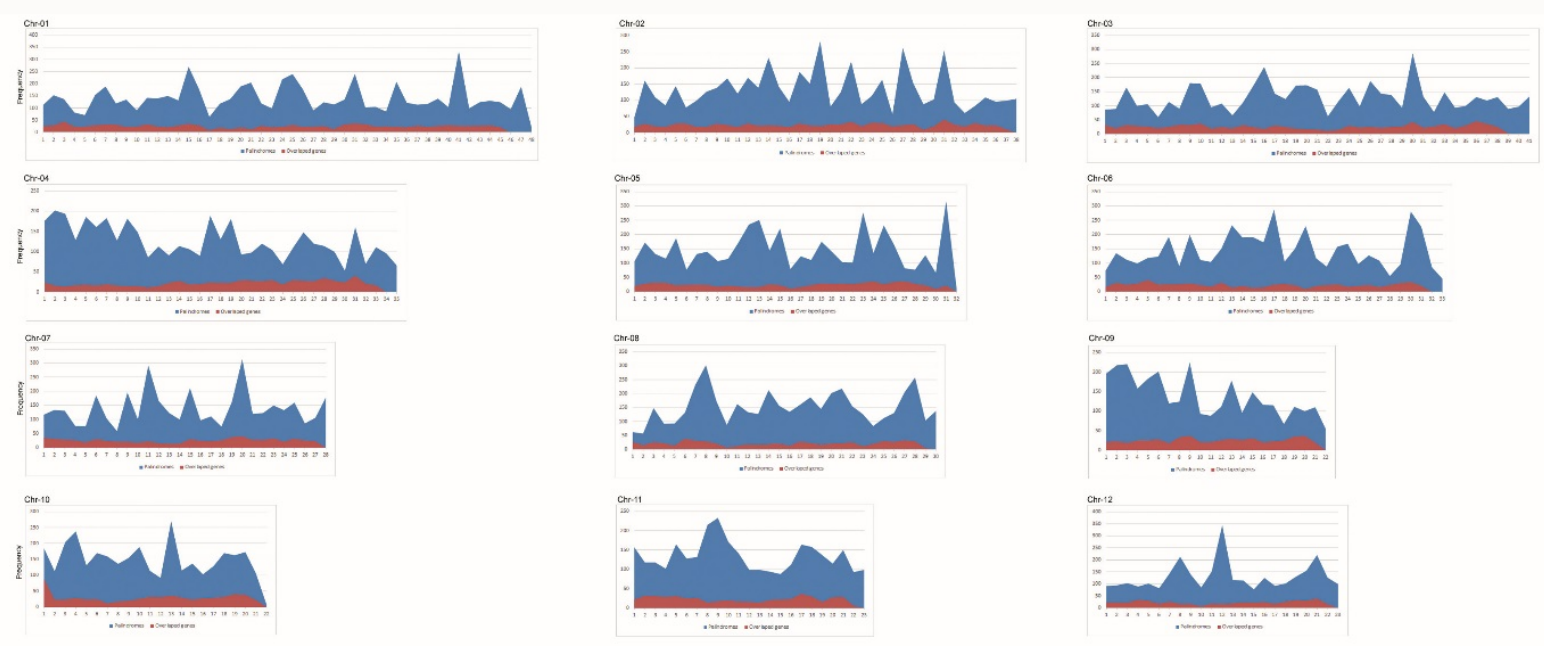

Figure 7: A graphical scheme showing the overlapping between palindrome regions and rice genes along each chromosome.

\section{Discussion}

In this research we succeeded to search and find many numbers of palindromic sequences in the rice ( $O$. sativa subsp. indica) genome. As bioinformatics analyses showed rice genome is occupied by nearly 51000 long palindrome regions, and these regions occupies more than $41 \%$ of the rice nuclear genome. In a research it was reported that only $5 \%$ of vertebrate mitochondrial DNA has palindrome sequences, and in contrast mammalian mitochondrial genome don't have palindrome sequences [20]. Regarding that these types of sequences are classified as repetitive elements, a study showed that around $51 \%$ of the rice genome is occupied by repetitive elements [3]. In other hand, transposable elements as a main class of repetitive sequences are virtually ubiquitous and make up, for instance, $20 \%$ of a typical D. melanogaster genome [17], $50 \%$ of a $\mathrm{H}$. sapiens genome [21], and $85 \%$ of a $Z$. mays genome [22]. Why repetitive sequences including transposons and palindromic regions have such an extended distribution in genomes, is probably related to their known or unknown functions in genome evolution. For example, repetitive extragenic palindromic sequences are DNA targets for insertion sequence elements in bacteria [23]. Palindromic sequences are known to have roles in DNA replication [24] and RNA transcription [25]. Also it has been reported that palindromes control gene expression through interaction with transcription factors, they maybe stabilize mRNA by inhibiting nuclease activity; they have been shown to involve in mRNA localization, and organisms use palindromes as markers for self-DNA and non-self-DNA; palindromes maybe prevent from the expression of foreign genes, and they are involved in correct intron splicing; furthermore, most palindromes act as recognition sites for DNAbinding proteins $[23,26]$. Palindrome sequence plays a critical role in human foamy virus (HFV) dimerization [27]. The analysis of repetitive elements also revealed that repetitive elements in human genome may
Results showed that the distribution pattern of palindromes in rice chromosomes is adequately diverse, so that different chromosomes contain a different compact indices of palindromes. For example chromosome 3 has 123 palindromes per Mbp, indicating that the chromosome is a euchromatic chromosome governed a low diversity during evolution. In contrast, chromosome 8 has a high compact index of 154 palindromes per Mbp, most probably due to the high mobility of transposable elements in this chromosome [28].

In general, we can classify rice palindrome regions into short $(<100$ bp) and long ( $>100 \mathrm{bp}$ ) palindromes. Results of our research showed that the detected palindrome regions have a different lengths, so that whole palindrome length ranged between 101 to $11574 \mathrm{bp}$ (with an average of $\sim 3030 \mathrm{bp}$ ), and stem lengths ranged between 20 to $1610 \mathrm{bp}$ (with an average of $\sim 33 \mathrm{bp}$ ), and loop lengths ranged between 61 to $10000 \mathrm{bp}$ (with an average of $2963 \mathrm{bp}$ ). As before notified these long palindromes are over-represented in the rice nuclear genome (nearly 51000 long palindromes). Similarly, [7] reported that while the short palindromes (2-12 bp) were under-represented, the palindromes longer than $12 \mathrm{bp}$ were over-represented in the yeast genome. Apparently, palindrome length plays a critical role in genome stability. Palindromic sequences have been tied to different genomic rearrangements in different organisms depending on the length of the repeated sequences, although such repeated sequences are usually short and present at several functionally important regions in the genome. However, long palindromic sequences in yeast (Saccharomyces cerevisiae) genome are a major source of genomic instability $[20,29,30]$. The palindrome-mediated genomic instability is 
believed to be due to cruciform or hairpin formation and subsequent cleavage of this structure by structure-specific nucleases. Shorter palindromic sequences (shorter than $30 \mathrm{bp}$ ) are very stable while long palindromic sequences ( $>50 \mathrm{bp}$ ) generate double-strand breaks (DSBs) at a high frequency during meiosis in the yeast (Saccharomyces cerevisiae) that are not stable in vivo [29]. These sequences also increase inter- and intra-chromosomal recombination between homologous sequences. Hairpin structures can form from palindromic sequences due to base pairing in single-stranded DNA. These structures can be substrates for structure-specific nucleases and repair enzymes which can lead to a double-strand break (DSB) in the DNA. This then leads to loss of genomic material which can cause meiotic recombination $[20,29,30]$. Studies with a 140-bp long mutated palindromic sequence inserted in yeast have shown to lower postmeiotic segregation and increase rate of gene conversions, while shorter sequences do the opposite [31].

Correlation analysis showed that palindrome number plays a significant role in the expansion of chromosome size (R2>92\%; Figure 4 ), although palindrome length has a relatively high negative effect on chromosome size; that is, the longer palindromes can be found in shorter chromosomes. This reverse effect causes a balanced occupation rate in different rice chromosomes, so that except for chromosomes 2 and 3, more than $40 \%$ of chromosome lengths are occupied by palindromic sequences (see Table 1). To verify this hypothesis, we calculated a more sensible index named PL:CL ratio (Figure 8 ). To do this, we divided average palindrome length (PL) of each chromosome by the respective chromosome length (CL). As seen in the figure, this ratio well explains (R2>90\%) the negative effect of palindrome length on chromosome expansion. Piegu et al. [32] concluded that accumulation of repetitive elements, particularly retrotransposable elements, besides polyploidy, is the main factor of genome size increase in higher eukaryotes. They showed that the genome of Oryza australiensis, a wild relative of the Asian cultivated rice (O. sativa), has accumulated more than 90,000 retrotransposon copies during the last three million years, leading to a rapid twofold increase of its size.

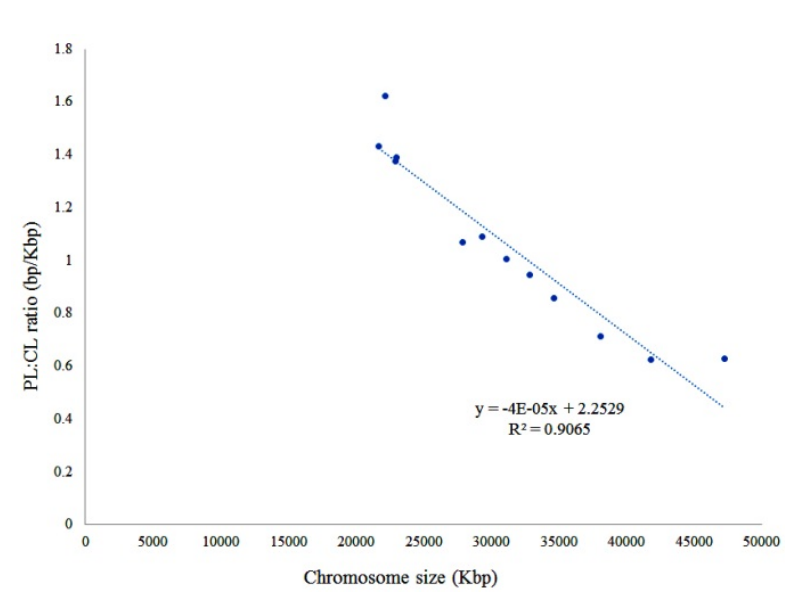

Figure 8: Relationship of PL:CL ratio on chromosome size in rice. PL: Palindrome Length; CL: Chromosome Length.

Results of our research also showed that one palindrome sequence occur every 7.3 Kbp in the rice genome, although these characteristics differed between chromosomes and even between the genomic locations. This value is considerably lower than that of (18.8 Kbp) reported for simple sequence repeats (SSR) in the rice genome [33]. These findings show that rice genome is rich in the palindrome sequences. A simulation study showed that there are 100 palindromes in every 1000 base pairs of a randomly generated sequence [4].

GC content analysis showed that average GC content of all detected whole-length palindromes is $42.1 \%$, with being $40.9 \%$ and $42.0 \%$ the GC contents of stems and loops, respectively, indicating that the long palindrome sequences are GC-poor, although a considerable number of palindromes doesn't follow this general rule (Figure 5). In fact, $13.4 \%$ and $8.5 \%$ of palindromes are GC-rich in stems and loops sections, respectively. In contrast, $52.1 \%$ and $39.5 \%$ of palindromes are AT-rich in stems and loops sections, respectively (Table 3). These results show the low-complexity of palindrome regions in the rice genome. Similarly, Sheari et al. [30] reported that there was a large chance of finding a palindrome in low complexity sequences. Many low-complexity regions are highly unstable due to the action of replication slippage and recombination [34,35] and the uncontrolled expansion of short sequence motifs causes several human diseases, such as Huntington's disease and other neurodegenerative disorders [34]. A hypothesis is that these regions increase phenotypic variation within populations, facilitating adaptation [36]. An alternative hypothesis to explain the abundance of low-complexity regions is that they facilitate the formation of novel coding sequences $[37,38]$. Another finding of our research is that palindromic regions of each chromosome have overlaps with $20.5 \%$ of the genes in the same chromosome, and that distribution patter of the overlapping genes is more concentrated in chromosomal arms which are gene-rich regions. Jiang et al [39] found that MITEs are mainly distributed in the chromosomal arms, and in contrast Feng et al [40] reported that retrotransposons are concentrated in the heterochromatic regions around the centromeres. Lisnić et al. [7] reported that long palindromes in yeast (Saccaromyses cervisiea) genome mainly locate in intron regions of genes.

In addition to the possible application of data obtained by palindrome identification in the development of a marker system for the use in some genetic studies such as evaluation of genetic variation and gene mapping $[13,14]$ these findings can be serve as a useful tool in population structure analysis and genome evolution studies.

\section{Conclusion}

Based on the results of this research it can be concluded that the rice genome is rich in long palindromic sequences that triggered most variation during evolution. Generally, both sections of palindromic sequences including stems and loops are AT-rich, indicating that these regions locate in the low-complexity segments of the rice chromosomes. The palindrome identification can help to develop a molecular marker system facilitating some genetic studies such as evaluation of genetic variation and gene mapping and also serves as a useful tool in population structure analysis and genome evolution studies. With the method of extracting rice palindrome regions in this research we will be able to extract and characterize these types of sequences in any other organism. Next research can be focused on the development of a simple, PCR-based marker system for detecting polymorphisms in palindromic regions for subsequent applications.

\section{Acknowledgments}

We thank Shahid Beheshti University for annual grants and University of Zanan for partial financial support of the work. 


\section{References}

1. Juretic N, Bureau TE, Bruskiewich RM (2004) Transposable element annotation of the rice genome. Bioinformatics 20: 155-160.

2. Yuan Q, Ouyang S, Liu J, Suh B, Cheung F, et al. (2003) The TIGR rice genome annotation resource: annotating the rice genome and creating resources for plant biologists. Nucleic Acids Res 31: 229-233.

3. Horng JT, Huang HD, Jin MH, Wu LC, Huang SL (2002) The repetitive sequence database and mining putative regulatory elements in gene promoter regions. J Comput Biol 9: 621-640.

4. Ninh A, Battig L (2012) Discovering the distribution of palindromic sequences in the SMAD4 gene using large and medium deletions and the resulting RNA structure predictions. J Exp Second Sci 2: 1-7.

5. Rattray AJ (2004) A method for cloning and sequencing long palindromic DNA junctions. Nucleic Acids Res 32: e155.

6. Nakazono M, Kanno A (1994) Palindromic repeated sequences (PRSs) in the mitochondrial genome of rice: evidence for their insertion after divergence of the genus Oryza from the other Gramineae. Plant Mol Biol 24: 273-281.

7. Lisnić B, Svetec IK, Sarić H, Nikolić I, Zgaga Z (2005) Palindrome content of the yeast Saccharomyces cerevisiae genome. Curr Genetics 47: 289-297.

8. Araya CL, Payen C, Dunham MJ, Fields S (2010) Whole-genome sequencing of a laboratory evolved yeast strain. BMC Genomics 11: 88 .

9. Brewer BJ, Payen C, Raghuraman MK, Dunham MJ (2011) Origindependent inverted-repeat amplification: A replication-based model for generating palindromic amplicons. PLoS Genetics 7: e1002016.

10. Tee SH (2013) Pattern analysis of tandem repeats in Nlrp1. Cornel Univ Library 1302: 2041.

11. Gemayel R, Vinces MD, Legendre M, Verstrepen KJ (2010) Variable tandem repeats accelerate evolution of coding and regulatory sequences. Ann Rev Genetics 44: 445-477.

12. Xu W, Liu L, Emson PC, Harrington CR, Charles IG (1997) Evolution of a homopurine-homopyrimidine pentanucleotide repeat sequence upstream of the human inducible nitric oxide synthase gene. Gene 204: 165-170.

13. Dong W, Shu-Xia S, Fang-Yuan G, Xian-Jun L, Zhi-Hua L, et al. (2009) Mapping a rice glabrous gene using simple sequence repeat markers. Rice Science 16: 93-98.

14. Ahmadikhah A (2013) Advanced Genetics: Publication of Gorgan Univ Agricultural Sciences and Natural Resources, Gorgan, Golestan 69-78.

15. Yang G (2001) Kiddo: A new transposable element family closely associated with rice genes. Mol Genet Genomics 266: 417-424.

16. Rao RS (2010) DNA repetitive sequences-types, distribution and function: A review. J Cell Mol Biol 7: 1-11.

17. Bergman CM, Quesneville H, Anxolabehere D, Ashburner M (2006) Recurrent insertion and duplication generate networks of transposable element sequences in the Drosophila melanogaster genome. Genome Biol 7: R112.

18. Dyer R (2009) Biological data analysis using R. Department of Biology Center for the Study of Biological Complexity. Virginia Common Wealth Univ 226.

19. Wittelsbürger U, Pfeifer B, Lercher MJ (2015) WhopGenome: high-speed access to whole-genome variation and sequence data in R. Bioinformatics 31: 413-415

20. Arunkumar K, Nagaraju J (2006) Unusually long palindromes are abundant in mitochondrial montrol regions of insects and nematodes. Plos One 1: e110.

21. Lander ES, Linton LM, Birren B, Nusbaum C, Zody MC, et al. (2001) Initial sequencing and analysis of the human genome. Nature 409: 860-921.
22. Schnable PS, Ware D, Fulton RS, Stein JC, Wei F, et al. (2009) The B73 maize genome: complexity,diversity,and dynamics. Science 326 1112-1115.

23. Tobes R, Pareja E (2006) Bacterial repetitive extragenic palindromic sequences are DNA targets for insertion sequence elements. BMC Genomics 7: 62 .

24. Willwand K, Mumtsidu E, Kuntz-Simon G, Rommelaere J (1998) Initiation of DNA replication at palindrom ic telomeres is mediated by a duplex-to-hairpin transition induced by the minute virus of mice nonstructural protein NS1. J Biol Chem 273: 1165-1174.

25. Chu WM, Ballard RE, Schmid CW (1997) Palindromic sequences preceding the terminator increase polymerase III template activity Nucleic Acids Res 25: 2077-2082.

26. Giel-Pietraszuk M, Hoffmann M, Dolecka S, Rychlewski J, Barciszewski J (2003) Palindromes in proteins. J Protein Chem 22: 109-113.

27. Cain D, Erlwein O, Grigg A, Russell RA, McClure MO (2001) Palindromic sequence plays a critical role in human foamy virus dimerization. J Virol 75: 3731-3739.

28. Bigot Y, Hamelin MH, Periquet G (1990) Heterochromatin condensation and evolution of unique satellite-DNA families in two parasitic wasp Species: Diadromus pulchellus and Eupelmus vuilleti (Hymenoptera). Mol Biol Evol 7: 351-364

29. Nasar F, Ankowskic J, Nag DK (2000) Long palindromic sequences induce double-strand breaks during mmeiosis in yeast. Mol Cell Biol 20: 3449-3458.

30. Sheari A, Kargar M, Katanforoush A, Arab S, Sadeghi M, et al. (2008) A tale of two symmetrical tails: Structural and functional characteristics of palindromes in proteins. BMC Bioinformatics 9: 274.

31. Nag DK, Kurst A (1997) A 140-bp-long palindromic sequence induces double-strand breaks during meiosis in the yeast Saccharomyces cerevisiae. Genet 146: 835-847.

32. Piegu B, Kim H, Guyot R, Collura K, Picault N, et al. (2006) Doubling genome size without polyploidization: dynamics of retrotranspositiondriven genomic expansions in Oryza australiensis a wild relative of rice. Genome Res 16: 1262-1269.

33. Li C, Zhang Y, Ying K, Liang X, Han B (2004) Sequence variations of simple sequence repeats on chromosome- 4 in two subspecies of the Asian cultivated rice. Theor Appl Genet 108: 392-400.

34. Ellegren H (2004) Microsatellites: simple sequences with complex evolution. Nat Rev Genetics 5: 435-445.

35. Gatchel JR, Zoghbi HY (2005) Diseases of unstable repeat expansion: mechanisms and common principles. Nat Rev Genetics 6: 743-755.

36. Kashi Y, King DG (2006) Simple sequence repeats as advantageous mutators in evolution. Trends Genet 22: 253-259.

37. Green H, Wang N (1994) Codon reiteration and the evolution of proteins. Proc Natl Acad Sci 91: 4298-4302.

38. Toll-Riera M, Rado-Trilla N, Martys F, Alba MM (2012) Role of lowcomplexity sequences in the formation of novel protein coding sequences. Mol Biol Evol 29: 883-886.

39. Jiang N, Feschotte C, Zhang X, Wessler SR (2004) Using rice to understand the origin and amplification of miniature inverted repeat transposable elements (MITEs). Curr Opin Plant Biol 7: 115-119.

40. Feng Q, Zhang Y, Hao P, Wang S, Fu G, et al. (2002) Sequence and analysis of rice chromosome. Nature 420: 316-320. 